\title{
Silicon integrated nanophotonics: from fundamental science to manufacturable technology (Presentation Video)
}

\section{Yurii Vlasov}

Yurii A. Vlasov, "Silicon integrated nanophotonics: from fundamental science to manufacturable technology (Presentation Video)," Proc. SPIE 9367, Silicon Photonics X, 93671X (23 April 2015); doi: 10.1117/12.2197116

SPIE. Event: SPIE OPTO, 2015, San Francisco, California, United States 


\title{
Silicon integrated nanophotonics: from fundamental science to manufacturable technology (Presentation Video)
}

\author{
Yurii A. Vlasov, IBM Thomas J. Watson Research Center (USA)
}

\begin{abstract}
The IBM Silicon Nanophotonics technology enables cost-efficient optical links that connect racks, modules, and chips together with ultralow power single-die optical transceivers. I will give an overview of its historical development, technology differentiators, current status and a roadmap.
\end{abstract}

View presentation video on SPIE's Digital Library: http://dx.doi.org/10.1117/12.2197116.4093587678001 